\title{
Intelligence Quotient in Children with Hypermetropia
}

\author{
Dr. Viji Devanand ${ }^{1}$, Dr. S. U. Chithrapavai ${ }^{2}$ \\ ${ }^{1}$ Associate Professor, Department of Physiology, Stanley Medical College, Chennai. \\ ${ }^{2}$ Post Graduate, Department of Physiology, Stanley Medical College, Chennai.
}

\begin{abstract}
Refractive errors are a common worldwide problem. Several hypotheses explain a relationship between hypermetropia and intelligence. This study is done to study the Intelligence Quotient in school children with hypermetropia and compare that to children with normal vision. This study was done in Department of Physiology in subjects attending the Ophthalmology Out Patient Department, Department of Ophthalmology, Stanley Medical College, Chennai. 30 subjects of age group 8 to 13 years were chosen and IQ test was performed using Binet- Kamath test, and compared with 30 control group chosen from Master Health Check up Programme. With this data obtained, statistical analysis was done using independent Student $t$ - test and chi square test using SPSS version 15. Of the 30 controls, 8 children were in grade I, 9 in grade II+, 8 in grade II, and 5 in grade III+. From 30 subjects, 6 children were in grade I, 5 in grade II+, 2 in grade II, 8 in III+, and 9 in grade IV with high significant $P$ value. From this study we conclude that hypermetropia has a direct influence on an individual's intelligent quotient and show that blurred hypermetropia may be a risk factor for lower $I Q$.
\end{abstract}

Keywords: intelligence quotient, hypermetropia, Binet- Kamath test

\section{Introduction}

Refractive errors are a common worldwide problem. Several hypotheses explain a relationship between hypermetropia and intelligence. Hypermetropic individuals seem to have lower IQ than normal individuals. Studies have also reported that hypermetropic children, regardless of their IQ have less school achievements. The correlation between severity of hypermetropia and low IQ has been shown to exist from various studies. ${ }^{[1]}$

\section{Aim}

1. Objective: To study the Intelligence Quotient in school children with hypermetropia and compare that to children with normal vision, i.e. emmetropics.

2. Materials and methodology:

Sample size: 30

Exclusion criteria: Children with ophthalmic disorders like myopia, squint, astigmatism, other systemic illness, previous ophthalmic surgical corrections.

Parameters studied: Intelligence Quotient using Binet- Kamath test.

Methodology: This study was done in Department of Physiology in subjects attending the Ophthalmology Out Patient Department, Department of Ophthalmology, Stanley Medical College, Chennai. After providing proper information and explanation to the study group written consent was obtained from the parent or the guardian of the subject who accompanied them to the OPD. A proper history including personal history, family history, past history of ophthalmic illness, refractory error corrections, spectacle usage was recorded.

30 subjects of age group 8 to 13 years were chosen and the study was conducted after recording their refractory error using Snellen's chart. The study individual were taken to a quiet room with good ambience and when the individual was comfortable with their parent or guardian nearby, IQ test was performed using BinetKamath test, and compared with 30 control group chosen from Master Health Check up Programme. BinetKamath test consists of a series of questionnaire from A to E, wherein each series consists of 12 questions. When the series was given as an individual test, the person recording results enters on the form the number of each piece pointed to. If the subject points to more than one piece, the piece he or she finally points to counts right or wrong. Right and wrong answers given by the study group was noted and an evaluation of results was done according to the tables of percentile norms provided along with the questionnaire ${ }^{[1]}$. It is graded as I, II+, II, III+, III, III-, IV, IV- and V, which is defined as,

Grade I: Intellectually superior, if the score lies at or above $95^{\text {th }}$ percentile point of his or her age group. Grade II+: Definitely above average in intellectual capacity, if the score lies at or above $90^{\text {th }}$ percentile point of his or her age group. 
Grade II: Definitely above average in intellectual capacity, if the score lies at or above $75^{\text {th }}$ percentile point of his or her age group.

Grade III+: Average in intelligence, if the score is greater than the median or $50^{\text {th }}$ percentile point of his or her age group.

Grade III: Intellectually average, if the score lies between $25^{\text {th }}$ and $75^{\text {th }}$ percentile point of his or her age group.

Grade III-: Intellectually average, if the score is less than $50^{\text {th }}$ percentile point of his or her age group.

Grade IV: Definitely below average in intellectual capacity, if the score lies at or below $25^{\text {th }}$ percentile point of his or her age group.

Grade IV-: Definitely below average in intellectual capacity, if the score lies at or below $10^{\text {th }}$ percentile point of his or her age group.

Grade V: Intellectually impaired, if the score lies at or below $5^{\text {th }}$ percentile point of his or her age group ${ }^{[1]}$.

The most satisfactory method of interpreting the significance of a person's total score is to consider it in terms of the percentage frequency with which a similar score is found to occur amongst people of his or her own age. This has the advantage over other methods that no priori assumption is made that in childhood the development of intellectual capacity is necessarily uniform or disturbed symmetrically. For practical purposes it is convenient to consider certain percentages of the population and to group people accordingly. In this way it is possible to classify a person according to the score he or she obtains. With this data obtained, statistical analysis was done using independent Student t- test and chi square test using SPSS version 15.

\section{Results}

Table 1: Showing mean and standard deviation of control and study group:

\begin{tabular}{|l|l|l|l|}
\hline Group & $\mathrm{N}$ & Mean \pm SD & P value \\
\hline Controls & 30 & $80.67 \pm 15.96$ & \multirow{2}{*}{$<0.001^{* *}$} \\
\hline Subjects & 30 & $59.83 \pm 28.84$ & \\
\hline
\end{tabular}

** $\mathrm{P}$ value $=<0.010$ highly Significant

Table 2: Showing grading of control and study group:

\begin{tabular}{|c|c|c|c|c|c|}
\hline Grade & Controls & Percentage & Subjects & Percentage & $\mathrm{P}$ value \\
\hline I & 8 & $26.7 \%$ & 6 & $20.0 \%$ & \multirow{9}{*}{$0.005 * *$} \\
\hline IIt+ & 9 & $30.0 \%$ & 5 & $16.7 \%$ & \\
\hline II & 8 & $26.7 \%$ & 2 & $6.7 \%$ & \\
\hline III+ & 5 & $16.7 \%$ & 8 & $26.7 \%$ & \\
\hline III & 0 & 0 & 0 & 0 & \\
\hline III- & 0 & 0 & 0 & 0 & \\
\hline IV & 0 & 0 & 9 & $30.0 \%$ & \\
\hline IV- & 0 & 0 & 0 & 0 & \\
\hline $\mathrm{V}$ & 0 & 0 & 0 & 0 & \\
\hline
\end{tabular}

$* * \mathrm{P}$ value $=<0.010$ highly Significant

\section{Discussion}

Objective of this study was to study intelligence quotient in children with hypermetropia. From table 2, of the 30 controls, 8 children were in grade I, 9 in grade II+, 8 in grade II, and 5 in grade III+. From 30 subjects, 6 children were in grade I, 5 in grade II+, 2 in grade II, 8 in III+, and 9 in grade IV with high significant P value.

Health can affect intelligence in various ways. Conversely intelligence can affect health. Health effects on intelligence have been described as being among the most important factors in the origins of human group differences in IQ test scores and other measures of cognitive ability. Several factors can lead to significant cognitive impairment, particularly if they occur during childhood when the brain is growing and blood brain barrier is less effective. Several studies have found hypermetropia to be associated with lower IQ and school achievements. Regarding the relationship to IQ, several explanations have been proposed one is that the hypermetropic child is less adapted at reading nearby distances and reads and studies less, which decreases intelligence. Another is that the hypermetropic children have a disadvantage at IQ testing which is near work because of more eye strain. Still another explanation is that pleiotropic gene affects the size of both brain and eyes simultaneously.

At age 11, those with hypermetropia had slightly reduced performance IQ in comparison with children without refractive error. The differences in performance IQ were attributable directly to the hypermetropia whereas the differences in verbal IQ were not attributable due to hypermetropia.

Far sightedness most commonly starts in early childhood. Normal development and lengthening of the eyeball occurs during early childhood. Eyes remain slightly farsighted when the eye has finished growing around 9 years of age, but they usually make up for the remaining refractive error, allowing the child to see well. In a study conducted by Sheila, it was quoted that," Children from a population sample whose cycloplegic 
refractive errors included myopia, pre-myopia and hypermetropia were compared on measures of IQ and reading with a group of children without significance refractive errors. At age 11 both those with myopia and with pre-myopia had increased verbal and performance IQ, while those with hypermetropia had slightly reduced verbal and performance IQ, in comparison with the children without refractive errors. The differences in verbal IQ were not attributable simply to earlier differences, but the differences in performance IQ were attributable to earlier differences" ${ }^{[2]}$. In a study conducted by Sandfeld et al, they had concluded that children with any ocular disorders, like hyperopia, astigmatism, anisometropia, and strabismus and contrast sensitivity were related to low IQ ${ }^{[3]}$.

When a study was conducted between children from mainstream school and children from school of the learning disabled, both groups presented with different visual deficiencies. However in that study it was noted that both groups have visual deficiencies which can impact negatively on their learning skills. Other factors in this study, for example, social and cultural backgrounds, poor responses from lack of understanding of the tests, or learning problems that were not evaluated prior to the visual evaluations could have also contributed to the findings. Based on that study it was concluded that, it is important that full and proper visual screenings to be conducted at schools, irrespective of whether the school is a mainstream or school for the learning disabled ${ }^{[4]}$. Another study conducted by raven et al, matrices was administered to 356 students of 5 th and 6 th grades to determine the effects of past experiences related to race, social class, and sex. They observed that all social class differences were significant, while a racial difference was found only in the lower class and no sex differences were significant. They concluded that future research must consider race, class, and sex in its investigation of the usefulness of the matrices, and, more generally, experiential influences on intelligence and school achievement $^{[5]}$.

\section{Conclusion}

There is evidence to suggest that hypermetropia is a hereditary condition. But, the recent rapid upsurge of hypermetropia implies that environmental factors may also contribute in the development of hypermetropia in genetically predisposed individuals. From this study we conclude that hypermetropia has a direct influence on an individual's intelligent quotient and other recent studies too show that blurred hypermetropia may be a risk factor for lower IQ as discussed.

\section{References}

[1] Standard Progressive Matrices, Sets A to E. Prepared by J.C.Raven.

[2] Refractory error, IQ and reading ability. Sheila M. Williams; Gerdon.F. Developmental Medicine and Child Neurology 1988 vol 30 page $735-742$.

[3] Hypermetropia is more common in low IQ children. I. Sandfeld; H. Jensen. Invest Ophthalmol Vis Sci 2004.

[4] Degree of hypermetropia in relation to intelligence and educational level. Eames Th. American journal Ophthalmol 1955; 39; 375377 .

[5] Social class, race, and sex differences on the raven (1956) standard progressive matrices. Tulkin, steven r.; newbrough, j. R. Journal of consulting and clinical psychology, vol 32(4), aug 1968, 400-406. 